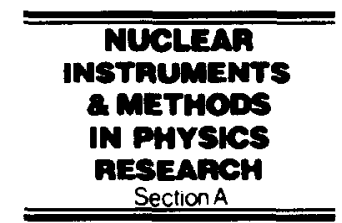

ELSEVIER

\title{
Review of the status of superconducting accelerator and detector magnets at extremely high fields ${ }^{1}$
}

\author{
R.M. Scanlan ${ }^{a}$, W.A. Barletta ${ }^{\text {a** }}$, D. Dell'Orco ${ }^{a}$, A.D. McInturff ${ }^{\text {a }}$, A. Asner ${ }^{\text {, }}$, \\ E.W. Collings ${ }^{c}$, P.F. Dahl ${ }^{d}$, H. Desportes ${ }^{d}$, A. Devred ${ }^{\text {e }}$, R. Garre ${ }^{f}$, E. Gregory ${ }^{\mathrm{g}}$, \\ W. Hassenzahl ${ }^{\text {h }}$, M. Lamm ${ }^{i}$, D. Larbalestier', D. Leroy ${ }^{\mathrm{k}}$, P. McIntyre', J. Miller ${ }^{\mathrm{m}}$, \\ T. Shintomi ${ }^{n}, H$. ten Kate ${ }^{o}, S$. Wipf ${ }^{p}$ \\ ${ }^{2}$ Lawrence Berkeley National Laboratory, Berkeley, CA 94720. USA \\ ${ }^{\mathrm{h}} 1261$ Genolier-VO. Switzerland \\ 'Department of Materials, Science and Engineering, The Ohio State University, Columbus, OH 43210, USA \\ ${ }^{\mathrm{d}}$ U.S. Department of Energy, Division of High Energy Physics, Washington, DC 20585, USA \\ ${ }^{\circ}$ CEA-Saclay, STCM, Gif-sur-Yvette, France \\ 'Europa Metalli-LMI, De Barga, Italy \\ "Intermagnetics General, Advanced Superconductors (IGC). Waterbury, CT 06704, USA \\ 'Piedmont, CA 94611. USA \\ 'Fermi National Accelerator Laboratory, Batavia, IL 60510, USA \\ ${ }^{\mathrm{A}}$ Applied Superconductivity, University of Wisconsin, Madison, WI 53706, USA \\ ${ }^{k}$ CERN, CH-12Il Geneva. Switzerland \\ 'Department of Physics, Texas A \& M University, College Station, TX 77843, USA \\ ${ }^{\mathrm{m}}$ National High Magnet Field Laboratory, Tallahasee. FL 32306. USA \\ "KEK, National Laboratory for High Energy Physics, Tsukuba, Japan \\ "Applied Physics-Low Temperature Division, Twente University, Enschede. The Netherlands \\ ${ }^{\top}$ Deutsches Elektron-Synchrotron (DESY), Hamburg, Germany
}

Received 13 March 1996

\section{Preface: Why very high fields magnets}

Probes of the high energy frontier of fundamental interactions of particles have become characterized by ever-increasing size and complexity. There are strong arguments that the ultimate energy potential of acceleratorbased high-energy physics will remain the province of proton supercolliders. Moreover, technical constraints (such as materials damage and radiation tolerance) will set definite limits on achievable and tolerable (in the detector) luminosity, limits that will decrease as the energy of the supercollider is increased. An illustration of the performance of present and proposed colliders appears in Fig. 1. While design characteristics of the Tevatron, LHC, the ill-fated SSC, and the proposed Eloisatron are all based on

\footnotetext{
${ }^{*}$ Corresponding author. Fax $+1510486 \quad 6003$, e-mail wabarletta@lbl.gov.

'Summary of 29th Workshop of the INFN Eloisatron Project,

"Ettore Majorana" Center for scientific Culture, Erice, Italy.
}

the use of ductile (NbTi) superconductor in the main accelerator dipoles and quadrupoles, the potential for upgrade of these machines depends on the practicality of increasing the dipole magnet field by a factor of 1.5 to 3 .

There is a common misperception in the high energy physics community, largely based on early SSC studies, that dipoles with fields $>8 \mathrm{~T}$ are not useful for supercol-

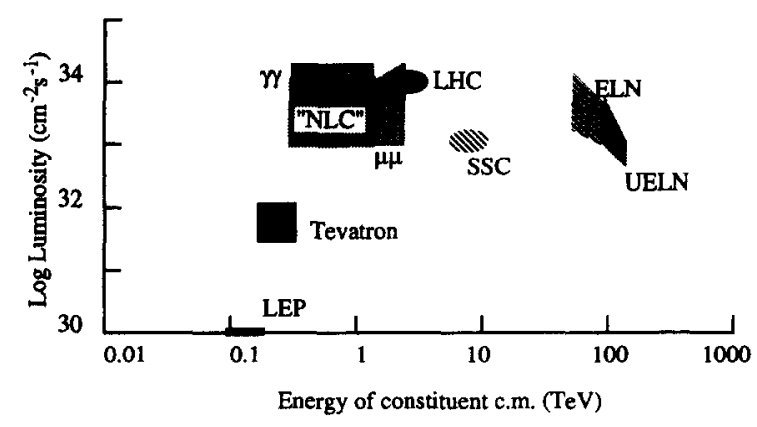

Fig. 1. Performance of present and proposed colliders. 
liders in that the use of higher fields will increase collider cost. This type of analysis ignores several important considerations.

1) The desirable field may be much higher than $8 \mathrm{~T}$ if the energy in an existing facility is to be maximized. Moreover, given the cost of a new supercollider and the logistics of setting up a new laboratory, the possibility of significant energy upgrade to an existing facility is highly desirable.

2) The total circulating charge in the beam of a collider increases inversely with $B$. Lower fields mean higher stored energy in the beam and require more powerful feedback systems and more stringent impedance requirements. The designs of beam aborts for the SSC and LHC at $400 \mathrm{MJ}$ of beam energy are problematic. Raising $B$ lowers both the demands on the feedback systems and on the abort systems.

3) The experience with the SSC suggests that the increase in extent of the physical infrastructure for a $50 \mathrm{~km}$ circumference makes both the technical sub-systems and the project management more complex.

4) As the accelerator circumference increases. the circulation frequency decreases. The Kolmogorov spectrum of the ground motion is a rapid inverse power of the frequency, so decreasing the site of the collider reduces its susceptibility to rapid emittance growth.

5) The optics design and achievable luminosity benefit from making the quadrupoles with as large a gradient as practical, especially in the interaction regions. The same technology that allows for dipoles of $15 \mathrm{~T}$ should allow for quadrupoles with gradients approaching $350 \mathrm{~T} / \mathrm{m}$.

For these reasons the development of a new generation of ultra-high field accelerator magnets needs to be a priority topic in the advance of accelerator technology.

\section{Introduction}

The physics performance of a particle accelerator is limited by energy and then by either beam intensity or by luminosity. In today's colliders, in which two counterrotating beams are made to intersect, these characteristics are determined by the physical size of the accelerator, the magnetic field, and the ability to accelerate and store a sufficient number of particles - usually protons. The development of superconductors and magnet designs adequate for these accelerators has a long and complicated history, the highlights of which we can barely touch on here.

\subsection{Background}

The year 1995 marks approximately 30 years since the advent of the earliest superconducting magnets for applications in high-energy physics, and, indeed, not many more years since the first practical Type II superconductor appeared on the scene [1]. First among the various pioneering superconducting devices were solenoids, with their concomitant stabilizing techniques, ranging from fully ("cryogenic") stabilized conductors to "intrinsically" stable, filamentary composites. (Filamentary, twisted superconductors were first convincingly proposed at an epochal summer study on superconducting devices and accelerators organized at Brookhaven National Laboratory in 1968 [2].) These early years also saw several innovative tape-wound superconducting quadrupole magnets (Brookhaven. 1965), the first of a long sequence of everlarger superconducting coils for bubble chambers (Argonne National Laboratory, 1968), and the first operational superconducting beam transport dipoles (Brookhaven, 1973, CERN, 1973 and Argonne, 1976).

By about 1970. the accelerator community also sensed the need for fast ramp superconducting accelerator magnets for the next generation of particle accelerators beyond the conventional (resistive) proton synchrotrons then on the drawing board in Europe (CERN) and the U.S. (Fermilab). This need led to the establishment in Europe of the Group for European Superconducting Synchrotron Studies (the GESSS collaboration of Rutherford Laboratory. Saclay, and Karlsruhe), for the purpose of jointly developing and exploiting ac magnet technology. GESSS resulted in the implementation of a number of innovative accelerator dipole designs in the sponsoring laboratories, but, unfortunately, proved premature with respect to the primary goal: the launching of an actual superconducting accelerator project [3].

The first effort toward demonstrating a working superconducting accelerator was ESCAR (Lawrence Berkeley Laboratory, 1974). Initially conceived as a test bed for a full-blown superconducting accelerator system, ESCAR was drastically scaled down in midstream to a test of the superconducting magnet system alone. As such. ESCAR did provide considerable operating experience with superconducting magnet and cryogenic systems. Next in the historical sequence came ISAbelle at Brookhaven, designed initially as a $200 \times 200 \mathrm{GeV}$ proton-proton collider. ISAbelle did not materialize due to several circumstances. Initially, it was plagued by excessive "training" of the full-length $(5 \mathrm{~m})$ dipoles; subsequently, a reconfigured version of the project (CBA) based on magnets wound from cable, rather than braid, was cancelled due to shifting priorities within the high-energy physics community. The machine was abandoned in 1983 in favour of an all-out push on the SSC.

As it turned out, the first true superconducting accelerator materialized at Fermilab in the waning days of the ISAbelle and the early phase of the CBA. Constructed in two stages as the fruit of a separate development effort launched at Fermilab ca. 1972, the Tevatron, a superconducting upgrade of the existing $500 \mathrm{GcV}$ proton synchrotron, was completed in 1983 [4]. Today's "standard" superconducting accelerator magnet, the collared cosine 
theta dipole wound from the ubiquitous Rutherford cable, is a legacy of the Tevatron project. Hard on the heels of Fermilab's collider came HERA in Hamburg, an electronproton collider incorporating a proton ring whose superconducting magnets are refined versions of the Tevatron dipoles and the cold iron CBA geometry.

Sadly, the next stage in superconducting collider development, the Superconducting Super Collider (SSC), was terminated well into its construction and after ten years of dedicated effort by the U.S. high-energy community [5]. The effort did, however, succeed by demonstrating the feasibility of accelerator dipoles which operate in the $6.5 \mathrm{~T}$ range and which meet the exacting machine specifications of multi- $\mathrm{TeV}$ colliders [6]. Hopefully, dipoles and quadrupoles of this general type will prove their worth in the Large Hadron Collider (LHC) at CERN by the end of the decade in the 8-9 $\mathrm{T}$ range [7].

\subsection{Future demands for high field magnets in particle accelerators}

Future use of particle accelerators will be for a variety of practical applications and for expanding the frontiers of particle physics. Here we address the demands for highfield magnets that will be driven by particle physics. Two conceptually different types of development can be envisioned. The first is for magnets that will be used to improve or augment existing facilities, the second is for new accelerator facilities. Examples of the former are: special magnets-such as high-field quadrupoles for FNAL, and high-field dipoles that could double the LHC energy. The proposed Eloisatron is the primary example of the second type [8]. An accelerator using high-field magnets to achieve the highest possible energy on the FNAL site is intermediate between these two categories.

The choice of the appropriate field for any future magnet system is driven by physics requirements, time frame for development, existing and projected material capabilities, magnet design, and the combination of program resources and magnet costs. Magnets with unique capabilities for specific applications that will enhance the performance of existing and often expensive accelerators are likely to push high-field magnet development in the short term. The cost of these magnets, though important, will not be a major design criterion because it will be a small fraction of the cost of the total accelerator. On the other hand, the need for large numbers of high-field magnets for new accelerators will require a thorough analysis of component and system costs.

Since existing accelerator magnets operate in the 5-7 T range, and $9 \mathrm{~T}$ magnets have been made for the $\mathrm{LHC}$, the appropriate field for future development should be well above this level. This workshop conducted a preliminary evaluation of existing magnet designs and materials to determine the highest field strength that could be achieved as an extrapolation of current technology. This analysis
Table 1

Specifications for $15 \mathrm{~T}$ magnet

\begin{tabular}{ll}
\hline Operating field $[\mathrm{T}]$ & 15 \\
Operating temperature $[\mathrm{K}]$ & 1.8 \\
Magnet bore $[\mathrm{mm}]$ ) & 40 \\
Dynamic aperture, radius [mm] & 10 \\
Field quality, $\Delta B / B_{0}$ & $<10^{-4}$ \\
Ramp time $[\mathrm{s}]$ & 1000 \\
Conductor $J_{\mathrm{v}}$, non-copper $\left[\mathrm{A} / \mathrm{mm}^{2}\right]$ & 1000 \\
\hline
\end{tabular}

suggests that a $15 \mathrm{~T}$ magnet could be constructed within the next 5 years. We believe that this would be an appropriate development goal for the near term and have developed a program plan for proceeding through the construction of a short-model $15 \mathrm{~T}$ dipole. We believe that an equivalent quadrupole could be constructed using the technology developed for the dipole.

There is reason to believe that superconducting material performance could be extended to achieve $20 \mathrm{~T}$ magnets in approximately 15 years. To that end, we have established a program based on present technology to develop and build a $15 \mathrm{~T}$ magnet in 5 years. The development of a $20 \mathrm{~T}$ magnet is more problematic and we propose a path of research and development that could lead to a first shortmodel $20 \mathrm{I}$ magnet in a 10 year time frame.

\subsection{Specifications for $15 \mathrm{~T}$ magnet}

The initial goal of the $15 \mathrm{~T}$ magnet program is the construction of a $1 \mathrm{~m}$ model accelerator dipole in the next 5 years.

The tentative specifications of this magnet are given in Table 1.

\subsection{Specifications for $20 \mathrm{~T}$ magnet}

The proposed $20 \mathrm{~T}$ magnet is a dipole, and the 10 year goal is a $1 \mathrm{~m}$ model. It is realized that this is a large step in the development of magnets. Magnets of intermediate field above the $15 \mathrm{~T}$ design are likely to be required along the path to the development of such a high field design. The tentative specifications for this magnet are given in Table 2.

Table 2

Specifications for $20 \mathrm{~T}$ magnet

\begin{tabular}{ll}
\hline Operating field $[\mathrm{T}]$ & 20 \\
Operating temperature $[\mathrm{K}]$ & 1.8 \\
Magnet bore $[\mathrm{mm}]$ & 40 \\
Dynamic aperture, radius $[\mathrm{mm}]$ & 10 \\
Field quality, $\Delta B / B_{0}$ & $<10^{-4}$ \\
Ramp time $[\mathrm{h}]$ & 1 \\
Conductor $J_{\mathrm{c}}$, non-copper $\left[\mathrm{A} / \mathrm{mm}^{2}\right]$ & $1000-1500$ \\
\hline
\end{tabular}




\section{Structure}

The development of future dipoles will be based in part on experience to date. One key issue has been the overall structure. Higher field magnets are expected to introduce new challenges.

\subsection{Dipoles to date}

In accelerator dipoles to date, the design of structural components has primarily addressed two key issues:

1) Exerting sufficient control of global winding deflections so that field quality is not impaired, and

2) Exerting sufficient control of local conductor motions that premature quenches are eliminated and reliability of operation is assured.

\subsection{New issues}

Points 1 and 2 remain as key issues. However, the challenge of successfully addressing both of the above issues becomes more difficult as the field strength increases $(20 \mathrm{~T}$ imposes a net force on the windings at least 4 times greater than the force in a $10 \mathrm{~T}$ magnet). Also, the options for addressing the second issue are further complicated by the more limited list of conductors available for the high fields and the need for special winding/processing techniques in their application.

The conductors available for application in the 10-20 T range can be degraded reversibly or irreversibly at modest levels of distortional strain (i.e. that part of the total strain which is not hydrostatic). This feature imposes on the overall magnet-structure design:

- Management of local stresses to the degree that the performance potential of the superconductor is not intolerably compromised or lost with cyclic operation.

This third issue forces consideration of whether the structural component should be distributed within the windings, placed on the outside of the winding pack, or some variant of these two possibilities. For some conductor options where damage may be irreversible at extremely small levels of distortional strain, it will be essential that there be a component of structure adjacent to the current carrying element itself and capable of shunting the local load around it. This component needs simultaneously to have high modulus, high strength, and a thermal expansion coefficient compatible with the superconductor. When the degradation with strain is reversible and gradual, the designer will have to evaluate carefully whether the potential degradation is excessive or can be reliably predicted in comparison to the effective reduction of winding-pack current density which would be required by inclusion of distributed structural elements. To put this issue in perspective, it should be noted that the local mean stress at some points in the winding will likely exceed 200 $\mathrm{MPa}$ in a $20 \mathrm{~T}$ design. Local stresses could easily exceed this level by a significant factor if care is not taken with the local stress management.

- Impregnation versus ventilation in the insulation matrix of the coil package.

A decision whether or not to impregnate the windings must be wcighed carefully. The process fits quite naturally with some conductor choices and winding techniques (e.g., the wind-react-impregnate process). It also has clear structural implications for the magnet design, as well as having an impact on the quality of electrical insulation, the character of disturbances to the windings, and the ability to stabilize against them. Impregnation can certainly help with the local management of stresses on strain-sensitive conductors. Potentially, it can also stop relative motion of conductors, thereby eliminating one source of disturbances that might lead to premature quenching. Traditionally, however, the latter has been either difficult to achieve (because of micro-cracking in the epoxy) or not entirely adequate, since it may still leave the possibility of global slippage of a winding block against structure, which can also quench the conductor. Typical design allowables for the impregnation layer are as follows: $\sim 400 \mathrm{MPa}$ for compression transverse to the layer, a very low value of $\sim 10 \mathrm{MPa}$ for tension, $\sim 30 \mathrm{MPa}$ for bond shear, and in the range of $\pm 0.3 \%$ strain parallel to the layer.

Heat transfer is a major problem with impregnated coils: heat generated by beam losses, synchrotron radiation, and ac losses must pass through the impregnated matrix, and could produce substantial temperature differentials. If a porous insulating matrix could be devised, helium could percolate within the coil package much as it does with B-stage impregnated NbTi coils. On the other hand, the design features that allow permeation of helium into the windings inevitably lead to local concentrations of stress on some combination of conductor, insulation, or distributed structure. Either the impregnated or the ventilated design could be successful with careful attention to the unique features of each.

\subsection{Type of magnet}

The type of magnet construction selected has an impact on how these four structural issues are addressed in detail [9]. Three construction types may be categorized [10]:

- Shell or minimum-volume types (including $\cos q$, intersecting ellipse, or similar variants),

- block or racetrack type, and

- other special designs created to accommodate a special class of conductors (e.g., tapes, new forms of high temperature superconductors, etc.), to address unique features of a particular type of accelerator, or some combination of these.

The shell-type winding may be the least flexible in terms of accommodating a wide variety of conductor geometries. Arguably, it may also present fewer options for incorporating a distributed structure, if needed. However, it does 
present a simpler external geometry (more rounded) that may have advantages for coupling to external structure. External structure for controlling deflections of the windings remains an essential need. Grading of shell-type windings can be accomplished with multiple shells, albeit with some loss of advantage in achieving minimum volume of conductor. Distribution of structure between shells may be sufficient to achieve the required level of stress management (especially if such designs are reoptimized with an emphasis on local stress management).

There is little experience with the other design types. However, the block-type designs appear to offer many options for both conductor grading and distribution of structure within the windings.

Among the special designs we have discussed, the "pipe" design [11] and the "parallel walls" design [12] were created primarily to accommodate the use of tape conductors, which tend to have highly anisotropic performance with respect to the relative directions of field and transport current. In addition, these designs offer novel approaches in handling or eliminating the need to preload the windings-approaches that may find application in another winding type.

\section{Protection}

\subsection{General considerations}

When an accelerator magnet quenches, the quench protection system triggers an active protection scheme for the magnet which forces a major portion of the coils to rapidly become resistive [13]. The stored energy of the magnet is completely absorbed in the quenching magnet. The technical constraints are given by the maximum hotspot temperature, the mean temperature and mean gradient in the coils, and the inter-turn voltage during the quench. Meanwhile the rest of the machine can be discharged at a lower rate by bypassing the quenching magnets with diodes. The aim is to avoid quenching the rest of the machine.

\subsection{Example: $L H C$ protection system}

Table 3 indicates a few parameters that have been obtained in a $10 \mathrm{~T}$ magnet system.

Table 3

Protection parameters in $10 \mathrm{~T}$ magnet system

\begin{tabular}{ll}
\hline Current $[\mathrm{A}]$ & 15000 \\
Inductance $[\mathrm{mH} / \mathrm{m}]$ & 5 \\
Conductor width $[\mathrm{mm}]$ & 17 \\
Hot-spot temperature $[\mathrm{K}]$ & 300 \\
Internal voltage $[\mathrm{V}]$ & 425 \\
Voltage to ground $[\mathrm{V}]$ & 1000 \\
\hline
\end{tabular}

The LHC will operate in superfluid helium and use a proven polymide conductor insulation scheme. Quenches will be spread by quench heaters located on the perimeter of the outer coil layer in a system designed for the quenching magnet to absorb the full magnetic energy.

\subsection{Hot spot temperature and copper current density}

The basic relationship between the hot spot temperature and the copper current density is of the type:

$\int_{0}^{\Delta t} j_{\mathrm{cu}}^{2} \mathrm{~d} t+j_{\mathrm{cu}}^{2} \frac{\tau}{2}=K\left(\frac{T}{T_{0}}\right)^{1 / 2}\left(1+\frac{1}{\alpha}\right)$

where $\tau$ is the decay time constant of the magnet, and $\Delta t$ is the time delay for detection, firing, and thermal diffusion into the magnet windings. The parameter $K$ depends on the conductor properties (specific heat, electrical resistivity), and $\alpha$ is the ratio $\mathrm{Cu} /$ non- $\mathrm{Cu}$ areas. The main parameters to control and therefore minimize the hot spot temperature are $\Delta t, j_{\mathrm{cu}}, \alpha$, and $\tau$. As a starting point of any design, it will be assumed that $\Delta t=100 \mathrm{~ms}, \tau=300 \mathrm{~ms}$. Preliminary examination indicates that the hot spot temperature could be kept below $600 \mathrm{~K}$ with a current density in the copper around $1400 \mathrm{~A} / \mathrm{mm}^{2}$.

\subsection{Stored energy of a $15 T$ dipole}

The stored energy of a $15 \mathrm{~T}$ dipole ( $50 \mathrm{~mm}$ bore) could be as large as $1.6 \mathrm{MJ} / \mathrm{m}$, depending on the design. Compared with that of $10 \mathrm{~T}$ magnets, the stored energy increases by a factor of 3 . In order to reduce the mean temperature of the coils, the energy should be distributed throughout the whole volume of conductor by quench heaters located in each layer. The temperature gradient between layers should stay below $10 \mathrm{~K}$, so that the interlayer shear stress does not exceed $3 \mathrm{MPa}$, unless the layers are equipped with low friction surfaces such that they can slide easily relative to one another.

\subsection{Interturn voltage}

It is proposed that the self-inductance of a dipole should be limited to approximately $5-10 \mathrm{H} / \mathrm{m}$ corresponding to $\sim 50-100$ turns/pole. To be consistent with the stored energy, and satisfy the low self-inductance requirement, leads to currents per turn close to $20 \mathrm{kA}$, resulting in the voltage of the quenched coils being in the range of $400 \mathrm{~V}$ (8-10 V/turn). In these preliminary estimates, a current density of $2000 \mathrm{~A} / \mathrm{mm}^{2}$ in the non-Cu area of the conductor has been assumed. Concerning the ground insulation, it is reasonable to limit the voltages to $\sim 1 \mathrm{kV}$. It appears then that it would be suitable to have operating currents in the range $20-30 \mathrm{kA}$ for a $15-20 \mathrm{~T}$ accelerator dipole. These muiti-10 kA currents would require develop- 
ment of new higher-current diodes which today operate only up to $15 \mathrm{kA}$.

\subsection{Quench heaters}

The conclusion for heater feasibility is that it is clearly possible, within the parameters of the present heater development, for photoetched polyimide/copper/stainless steel heaters. The geometry of such a heater would be one in which all turns, or at least most turns of all layers, are covered at least $10 \%$ by the heaters. The heater using 12 $\mu \mathrm{m}$ foil requires 12 to $32 \mathrm{~J} / \mathrm{m}$ to switch a superconducting winding normal in superfluid helium. Developments that would be useful but not critical are:

a) $6 \mu \mathrm{m}$ thick stainless steel foil,

b) new glue to withstand $5 \mathrm{kV}$ for $50 \mu \mathrm{m}$ of polyimide/ stainless steel sandwich,

c) thermal diffusion rate improvement of a factor of 2 (new filled polymide films).

\subsection{Computer codes and model instrumentation}

Computer codes exist to calculate the quench and stress development in a quenching magnet (QUABER [14]; ANSYS [15]). They need to be checked by suitable measurements on models equipped with spot heaters to check the coil heating characteristics, and strain gauges to check stress levels between layers and the strain history during thermal cycling, aging and power sequencing for correct input parameters.

\section{Conductor}

\subsection{General conductor requirements}

The conductor requirement for accelerator magnets at 15 $\mathrm{T}$ and above are very stringent. In order to produce these fields in an efficient manner, relatively high $J_{c}$ conductors must be obtained. A value of $J_{c}=1000 \mathrm{~A} / \mathrm{mm}^{2}$ in the superconductor at the operating field is generally accepted as a realistic starting point. This value is then diluted by a high conductivity normal metal matrix necessary for coil protection, insulation, space for cooling medium, and support structure. In terms of conductor choice, $\mathrm{NbTi}$ will be used at fields up to its practical limit, which is about 11 $\mathrm{T}$ at $1.8 \mathrm{~K}$, since all practical high-field superconductors except NbTi are brittle. This also implies that conductor grading will be used wherever possible and that NbTi will be pushed to the limit by means of ternary additions, which increase the upper critical field by about $1 \mathrm{~T}$.

All candidate conductors for use at higher fields are brittle materials. This means that special coil fabrication techniques must be used. This includes careful handling. limiting magnet prestress and minimizing the differential thermal expansion/contraction problems. A minimum re- quirement is that the material be able to withstand the Lorentz force when the coil is energized, and that the material be able to survive a tensile strain of $0.5 \%$ without permanent degradation. In addition, the critical currents of many conductors under consideration exhibit a strain sensitivity. The conductor most developed for use at fields above $12 \mathrm{~T}, \mathrm{Nb}_{3} \mathrm{Sn}$, shows a relatively high $J_{\text {. }}$ strain sensitivity. This is an intrinsic property of $\mathrm{Nb}_{2} \mathrm{Sn}$; however, some results indicate that certain conductor manufacturing approaches or conductor configurations produce conductors that are more sensitive to strain than others. Furthermore, the strain dependence is more pronounced at high fields. A primary driving force behind the interest in $\mathrm{Nb}_{3} \mathrm{Al}$ as an alternative to $\mathrm{Nb}_{3} \mathrm{Sn}$ is the lower strain dependence of $J_{c}$. This is especially important above $15 \mathrm{~T}$. However, this conductor is not developed commercially to the extent of $\mathrm{Nb}_{3} \mathrm{Sn}$, and in any event fabrication is more difficult. (See the $\mathrm{Nb}_{3} \mathrm{Al}$ section.)

In order to meet accelerator magnet requirements, the diamagnetic effect of the superconductor must be controlled (its value rendered small and predictable). Although multifilamentary $\mathrm{Nb}_{3} \mathrm{Sn}$ may have a geometric filament diameter of 2-5 $\mu \mathrm{m}$, the filaments grow together during the reaction and the effective filament size can become the size of the region containing the $\mathrm{Nb}_{3} \mathrm{Sn}$ filaments, i.e., the order of tens of microns. Magnetization effects can be corrected; however, simple, low-cost correction requires a filament size less than $50 \mu \mathrm{m}$ with a desirable value being around $5 \mu \mathrm{m}$.

Eddy current coupling can be present in Rutherford cables, depending on the resistance between strands and on operating conditions, such as current ramp rates. This coupling must be controlled and be reproducible. Too low a value of resistivity leads to heat deposition during ramping, and can lead to magnet quenching and can produce unwanted multipoles. Interstrand resistance must be controlled by strand coatings or by high resistance core strips in the cable to minimize these effects.

Strain limitations on materials other than NbTi typically lead to the use of a wind-and-react coil fabrication procedure, which means that insulation must withstand the reaction cycle, which is several hundred hours at $650^{\circ} \mathrm{C}$ for $\mathrm{Nb}_{3} \mathrm{Sn}$. This means that the cable insulation must be fiberglas or a ccramic matcrial. Fiberglas is abrasionsensitive, especially after the sizing is removed from the insulation. Ceramics, such as mica, have low thermal conductivity and also poor shear strength.

In order to use any of these conductors in accelerator magnets, the operating current must bc high enough to allow protection (see section on protection). This implies a conductor which is built up of multiple wires or tapes. The form most commonly used is the Rutherford cable, [16] which has advantages of flexibility, high aspect ratio, transposition, and relatively high packing factor, in addition to high operating current. Other configurations, such as tapes, need to be developed for use in accelerator 
magnets, and design features such as ends must be tailored to their use. As we push to higher fields, other conductor configurations may become important. Rutherford cable modifications with internal cores [17] for strength, reduction of strand/strand coupling, or improved interstrand resistance, may be required. The assumption is made that the minimum amount of $\mathrm{Cu}$ needed for stability and protection will be incorporated in each strand in a graded coil design.

\section{2. $\mathrm{Nb}_{3} \mathrm{Sn}$ status and prospects for improvement}

Piece length of $\mathrm{Nb}_{3} \mathrm{Sn}$ is all-important, as with any other practical conductor. Any increase in properties must be made without adverse effects on piece length. The other property which is intimately related to $J_{\mathrm{c}}$ is their "effective filament" diameter. Provided that the effective filament diameter is not required to be $<50 \mu \mathrm{m}$, the present state of the art for $J_{c}$ of $\mathrm{Nb}_{3} \mathrm{Sn}$ at $4.2 \mathrm{~K}$ is $1500 \mathrm{~A} / \mathrm{mm}^{2}$ at $12 \mathrm{~T}$ in the non-copper. These non-copper properties can also be produced at $14 \mathrm{~T}$ and $1.8 \mathrm{~K}$. It is obvious that to make workable magnets in the region 15-20 T, the $J_{c}$ in the non-copper must be increased significantly. This can be done in two main ways:

1) increasing the volume fraction of $\mathrm{Nb}$ and $\mathrm{Sn}$ in the non-copper area (i.e., increasing the cross section of $\mathrm{Nb}_{3} \mathrm{Sn}$,

2) increasing the flux pinning strength of the $\mathrm{Nb}_{3} \mathrm{Sn}$ itself.

Method 1 tends to have an adverse effect on other properties, such as "effective filament diameter," copper purity, etc. Method 2 has received little recent attention and is not well understood. Refining the grain size is the standard method of raising $J_{\mathrm{c}}$ in $\mathrm{Nb}_{3} \mathrm{Sn}$. It works at lower fields but its effectiveness at fields of $15 \mathrm{~T}$ and above is less certain. New strategies, such as the introduction of artificial pinning centers, are needed. By incorporating these improvements it is reasonable to expect properties such as $1500 \mathrm{~A} / \mathrm{mm}^{2}$ at $15 \mathrm{~T}$ and $1.8 \mathrm{~K}$ in the next few years. This would give $\sim 750 \mathrm{~A} / \mathrm{mm}^{2}$ at $18 \mathrm{~T}$ and $1.8 \mathrm{~K}$. The next step will be to attempt to double this $18 \mathrm{~T}$ value in the subsequent 5 years.

One factor that must receive more attention is the stress sensitivity of the various types of $\mathrm{Nb}_{3} \mathrm{Sn}$ that are available commercially. The view has been expressed that multifilamentary internal tin is much more sensitive than tape. The overall $J_{i}$ of material in tape form is high, since no bronze is present to create dead spaces. Therefore, it is possible to expect $J_{\mathrm{c}}$ values twice those of the multifilamentary material.

Although $\mathrm{Nb}_{3} \mathrm{Sn}$ was first developed many years ago, it has received very little financial support recently. The fusion community has expressed a need for a material with moderate $J_{c}$ and relatively low loss capability at $12 \mathrm{~T}$ for the ITER program. Moderate funding is expected to be available to expand manufacturing facilities for quantity production of $\mathrm{Nb}_{3} \mathrm{Sn}$ required for the $15 \mathrm{~T}$, and at least part of the $15 \mathrm{~T}$ and at least part of the $20 \mathrm{~T}$ accelerator magnets.

\section{3. $\mathrm{Nb}_{3}$ Al status and potential}

Several processing methods have been proposed for $\mathrm{Nb}_{3} \mathrm{Al}$ in order to avoid the limitations due to the fact that the stoichiometric A15 phase forms only at elevated temperatures. The most direct approach, which has not yet been extended to commercial practice, consists of meltquenching stoichiometric $\mathrm{Nb}-25 \% \mathrm{AI}$ and then subjecting the resulting bcc phase to a "low temperature" (800$900^{\circ} \mathrm{C}$ ) anneal to produce an ordered A15 phase. Another high temperature processing method, and one that is capable of yielding long continuous lengths of wire, is a powder-in-tube approach. After the components are drawn to near final size, the precursor powders are laser or electron beam heated to form the desired phase. The disadvantage of this method is that it produces a monofilamentary wire which lacks the copper stabilizer.

Other methods consist of blending elemental $\mathrm{Nb}$ and $\mathrm{Al}$ components at the nanometer level, and then converting the mixture to $\mathrm{A} 15 \mathrm{Nb}_{3} \mathrm{Al}$ by a moderate $\left(800-900^{\circ} \mathrm{C}\right)$ heat treatment. Three promising routes using this approach are being developed: (1) a $\mathrm{Nb}$ tube approach being developed by NRIM in Japan, (2) a mechanically alloyed method being developed by IGC-ASI in the U.S., and (3) a jelly-roll of $\mathrm{Nb}$ and $\mathrm{Al}$ foils first developed in Italy (CNEN/LMI) and more recently in Japan (Sumitomo and Hitachi). Although none of these efforts are producing material on a commercial scale, small quantities of wire with $J_{c}=800 \mathrm{~A} / \mathrm{mm}^{2}$ at $12 \mathrm{~T}$ are now available for model coil fabrication. This effort is being stimulated by the ITER fusion program, as a possible replacement for $\mathrm{Nb}_{3} \mathrm{Sn}$.

\subsection{Potential of HTS materials as accelerator conductors}

Critical current values in the best short samples of HTS already exceed $10^{3} \mathrm{~A} / \mathrm{mm}^{2}$ in fields out beyond $20 \mathrm{~T}$ at $4.2 \mathrm{~K}$. The essential issue for conductors is therefore to develop such values in long lengths, and in accelerator conductor forms. The best properties are presently obtained in high aspect ratio $(\sim 10: 1)$ monocore tapes which might be suitable for parallel wall magnets, while multifilamentary conductors suitable for other magnet designs have $J_{c}$ values perhaps a factor of 2 lower. All present HTS conductors are based on $\mathrm{Bi}_{2} \mathrm{Sr}_{2} \mathrm{CaCu}_{2} \mathrm{O}_{x}$ (BSCCO2212) or $\mathrm{Bi}_{2} \mathrm{Sr}_{2} \mathrm{Ca}_{2} \mathrm{Cu}_{3} \mathrm{O}_{x}$ (BSCCO-2223) and at the present time the $J_{\mathrm{c}}(4 \mathrm{~K})$ of $\mathrm{BSCCO}-2212$ is actually greater than that of BSCCO-2223, even though the $T_{\mathrm{c}}$ of the BSCCO-2223 phase is about $20 \mathrm{~K}$ higher $(\sim 110 \mathrm{~K}$ vs $\sim 85 \mathrm{~K}$ ). This apparently paradoxical difference emphasizes an important property of HTS materials in which they 
differ fundamentally from the low temperature superconductors (LTS). The key limit to $J_{c}$ in HTS in the low temperature limit is the connectivity of the polycrystalline filaments. Weakly coupled grain boundaries, cracks and imperfect local composition all contribute to making the current path through the conductor percolative. Thus the true local $J_{c}$ is often much higher than the average $J_{c}$. This is a vital fact for future accelerator use, since it means that there is great potential for further development of $J_{\mathrm{v}}$. A gross idea of their potential is obtained by noting that the $J_{c}$ of epitaxial BSCCO thin films can lie in the range of $10^{4}-10^{5} \mathrm{~A} / \mathrm{mm}^{2}$, some two orders of magnitude above the best present polycrystalline conductor-like forms.

Multifilament conductors using the Oxide-Powder-inTube (OPIT) process of both BSCCO compounds are being made in lengths exceeding $1 \mathrm{~km}$, with filament numbers up to about 250 and with diameters ranging upwards from $10 \mathrm{~mm}$. Conductors using the Metallic Precursor (MP) process can achieve diameters $<1 \mathrm{~mm}$ but with lower present $J_{\text {. }}$. Most conductors are high aspect ratio even when multifilament, but recent efforts in making round wire BSCCO-2212 conductors look promising. Even tapes can be twisted when round so as to produce short twist pitches in tape form.

The strain tolerance of present conductors is very similar to that of $\mathrm{MF} \mathrm{Nb}_{3} \mathrm{Sn}$ when tested in tension. However, the performance under cyclic stress under axial compression, or under transverse loading, is less well understood; there is also concern that irreversible damage occurs much more quickly than in $\mathrm{Nb}_{3} \mathrm{Sn}$. This could easily be a manifestation of the fact that the present conductors contain many cracks (which also limit $J_{\mathrm{c}}$ ) and that these propagate easily to produce progressively smaller limits on $J_{c}$.

Few cables have yet been made of HTS conductors, and their cabling performance is hard to assess. Conductors based on pure $\mathrm{Ag}$ matrices are unlikely to be suitable for accelerator use, and the strengthened matrices that are presently being developed will be vital. Whether large aspect ratio conductors can be cables is unclear, but the potential of round wire conductors can also be explored.

\section{Program plans}

\subsection{I5 T magnets}

\subsubsection{Aim of the program}

The field range up to $15 \mathrm{~T}$ can be considered as a reasonable extension of the existing accelerator magnet technology, in view of recent progress on doped $\mathrm{Nb}_{3} \mathrm{Sn}$ wires and of predictable advances in superconducting materials, notably in superfluid helium.

Present activities concern:

- Developments for LHC dipoles and quadrupoles in the

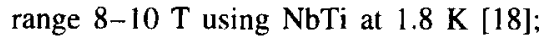

- a $\mathrm{Nb}_{3} \mathrm{Sn}$ dipole model which has produced $11.2 \mathrm{~T}$ at Twente University [19];

- the D20 dipole model at LBL aiming to $13 \mathrm{~T}$ with $\mathrm{Nb}_{3} \mathrm{Sn}$ at $4.3 \mathrm{~K}[20]$.

Other valuable experience is available from developments in high-field solenoids, such as those for very high field NMR applications and for the $45 \mathrm{~T}$ facility at the National High Magnetic Field Laboratory, Florida [21]. Fusion programs, particularly ITER, are also strong incentives for extremely high-field superconductors, al-

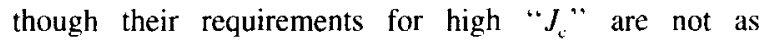
demanding as those for accelerator magnets.

\subsubsection{Conductor development}

The main requirements concern Al5 materials, $\mathrm{Nb}_{3} \mathrm{Sn}$ being the most extensively developed at present. However $\mathrm{Nb}_{3} \mathrm{Al}$ needs to be pursued in view of its lower transverse pressure sensitivity, as well as ternary alloys such as $\mathrm{NbTiTa}$ for grading purposes, if better than $\mathrm{Nb}_{3} \mathrm{Sn}$ in the graded region.

The general goal is for such conductors to increase the current density to the order of 1000 to $2000 \mathrm{~A} / \mathrm{mm}^{2}$ in non-Cu at operating field $(15 \mathrm{~T})$, with effective filament diameters as small as possible below the present $50 \mu \mathrm{m}$ common to internal diffusion processes in order to reduce magnetization effects. It is also important to minimize the strain sensitivity of $J_{\mathrm{c}}$ in view of large high field stresses.

Cable development follows wire production, with specific requirements concerning cabling degradation, mechanical behavior under combined stresses, ac losses, stability, quench behavior, etc. Particular cable structures should be investigated, such as cored cable, jacketed cable, coated strands, etc.

\subsubsection{Magnet design}

The main choice concerns the winding configuration, fulfilling the standard accelerator field requirements, and being compatible with other constraints, such as stress management, stability, losses, practical feasibility, and cost. At the $15 \mathrm{~T}$ field level, the cylindrical shell configuration, which is the universal scheme used in existing accelerator magnets, may still be the most practical structure. However, other configurations, such as racetrack block type coils, need to be further analyzed and compared with regard to performance and feasibility. In the case of the shell type configuration, a large number of parameters need to be optimized, and are subject to extensive design studies concerning mechanical and thermal behavior.

Technical areas covered in such studies are related to the mechanical support structure (external or distributed through the winding), the need and amount of prestress or preloading, the coil end design and support scheme, the insulation procedure, with or without full epoxy impregnation, depending on the wind-and-react sequence. Thermal requirements and many other fabrication items, such as conductor splices. instrumentation, and cooling chan- 
nels, influence the coil design. Since single bore and twin aperture dipoles have similar yoke dimensions, it is desirable in the case of single bore magnets to reduce the yoke size by using an active flux return configuration.

\subsubsection{Cooperative tasks}

In order to accomplish the 5-year scheduled development, a close inter-laboratory collaboration should be implemented as soon as possible, sharing test facilities (both existing ones and those needing upgrading) as well as design capabilities. Powerful computer codes are available for 2D and 3D analyses in all aspects of mechanical, electromagnetic and thermal calculations. ac losses, quench evolution, stability and protection. High-field test facilities are required for measuring $J_{c}$ vs field, temperature and strain conditions, as well as heat transfer and thermal calculations through complex geometries of cable, insulation, and structures. Sharing of tasks and fast distribution of results are essential to the rapid progress of the work.

\section{2. $20 \mathrm{~T}$ magnets}

\subsubsection{Aim of the program}

The field range between 16 and $20 \mathrm{~T}$ is very hard to reach with accelerator type dipole and quadrupole magnets without extensive research on materials, conductors and stress management. In particular, materials research to attain reasonable current densities in practical conductors at high levels of transverse stress is essential. To remain within the limits of what is practical and cost effective, it is believed that critical current densities in superconductors of about $2000 \mathrm{~A} / \mathrm{mm}^{2}$ are required - a factor 5 to 10 higher than is currently available in short pieces of experimental conductors.

It is the aim of the proposed program to set up a framework for such a development, by which material scientists are invited to contribute with new or improved materials, conductor $R \& D$ teams are invited to make practical and stress-resistant conductors of such materials, and magnet designers are invited to develop novel geometries for conductor and magnet construction schemes to handle and manage the stress levels in the magnet. Finally, the high energy physics community is invited to think about a future $20 \mathrm{~T}$ class of accelerators and to investigate proper specifications for such a magnet system.

\subsubsection{Magnet and conductor specifications}

The specifications for a $20 \mathrm{~T}$ dipole are driven by the requirements for reliable operation of an ultimate-energy collider:

- Central field $B_{0} \sim 20 \mathrm{~T}$;

- harmonic content $b_{n}<10^{-4} \mathrm{~cm}^{-n}$;

- dynamic range $B_{\max } / B_{\min }$ injection of at least $\sim 10: 1$;

- active quench protection with internal absorption of its stored energy.

These specifications result in some essential require- ments and/or limits on the superconductor and its stabilizer, the configuration of the superconductor into coil elements, the adequate support of coil elements in the coil, and the overall field design of the magnet.

\subsubsection{Superconductor}

The various field design concepts being developed for high-field dipoles require a high-field region which is several $(\sim 2-4)$ times the beam tube region (ca. $4-5 \mathrm{~cm}$ ) in each transverse direction. The total amp-turns per bore are thus $N I>2 B_{0}$ na/0-3 MA. Such an immense current block requires that the conductor package carry a large effective current density, even at this immense field strength. The magnet operation at $1.8 \mathrm{~K}$ has various important advantages with respect to the vacuum conditions over $4.2 \mathrm{~K}$ operation. Moreover the critical current density is higher at $1.8 \mathrm{~K}$, which roughly means a gain of about $2 \mathrm{~T}$. Therefore it is believed that a $20 \mathrm{~T}$ design should be based on supercritical helium. The most stringent requirement occurs for the inner coil elements, which must operate in the full central field. The winding package in these inner elements must sustain a mean critical current of at least $J_{\mathrm{c}}$ $\sim 500 \mathrm{~A} / \mathrm{mm}^{2}(20 \mathrm{~T}, 1.8 \mathrm{~K})$. Optimizing such designs gencrally will require $1000 \mathrm{~A} / \mathrm{mm}^{2}$ in the practical superconductor and a critical current density of 1500-2000 $\mathrm{A} / \mathrm{mm}^{2}$ in the unstrained basic superconducting material.

\subsubsection{High $T_{r}$ superconductors for $20 \mathrm{~T}$}

The upper critical fields of HTS conductors at liquid He temperatures are beyond the range of direct measurement and can be arrived at only by extrapolation. Thus, from a critical field standpoint, HTS materials should be ideal for the high-field parts of high-field magnets. The HTSC materials that are closest to commercialization in wire form are the two Bi-based materials Bi:2212 and Bi:2223. At present, most attention is being given to $\mathrm{Bi}: 2223$ for use at temperatures approaching $77 \mathrm{~K}$. Some interest is also being shown in $\mathrm{Bi}: 2212$, but in this case for use at $20-25$ $\mathrm{K}$ in association with cryo-cooler-type refrigeration. In summary, the present uses of both Bi:2212 and Bi:2223 require pushing the respective high-temperature limits of their ranges of applicability. However, accelerator magnets will always be operated in the liquid-He temperature range. Indeed, even within this range $1.8 \mathrm{~K}$ is preferable to $4.2 \mathrm{~K}$ Next, in addressing the use of Bi:HTS materials for accelerator magnets, important points can be made with regard to both stability and current density.

\subsubsection{Stability}

In the past, HTSC conductors have been criticized on the basis of low normal-zone propagation velocity. The existence of a "stagnant" normal zone offers the possibility of local burn-out unless a local quench can be detected in time for protection to be applied. But it must be remembered that this is a "high-temperature" (77 K) property associated with a correspondingly high heat 
capacity. At low temperatures $(<4.2 \mathrm{~K})$ the $\mathrm{Ag}$ (alloy)clad HTSC conductor has a low heat capacity and will behave much like a conventional superconductor with a correspondingly high normal-zone propagation velocity.

\subsubsection{Critical current densitv}

At the present time the $J_{\mathrm{c}}$ of HTS materials is limited, not so much by intrinsic intragrain $J_{c}$ as by the ability of the wire itself to carry such a $J_{c}$ over its entire length (i.e., to attain its true potential). This represents a processing difficulty and as such is being addressed within the framework of HTS wires for use at 20 and $77 \mathrm{~K}$, respectively. However, for low-temperature applications, opportunities exist for increasing the intragrain $J_{\mathrm{c}}$ by the inclusion of flux-pinning additions to the HTS formulations. Little attention is being paid to this scenario since it has been found that flux-pinning additions result in a lowering of $T_{c}$. Once the need for maximal $T_{c}$ is removed - as it is when 1.8-4.2 K operation is being considered the door is opened for the possibility of engineering

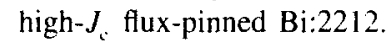

Finally, when considering high-field insert magnets using HTS superconductors, several other requirements demand attention. These are: 1) the development of very long length processing methods (e.g., continuous processing); 2) the further development of multifilament strands and their assembly into cables; 3 ) the inclusion of mechanical reinforcement in the HTS strand to provide mechanical protection, both during processing and afterwards in service.

\subsubsection{Stabilizer}

The conductor must be stabilized sufficiently to suppress micro-quenches (training), and to provide a realistic strategy for quench protection. These considerations require that the conductor contain sufficient high-conductivity stabilizer for a current density during a quench of $\sim 1500 \mathrm{~A} / \mathrm{mm}^{2}$ in the copper. The effective current density in a cable element would then be $J_{\mathrm{c}} \sim 375 \mathrm{~A} / \mathrm{mm}^{2}$ overall for $J_{\mathrm{c}}=500 \mathrm{~A} / \mathrm{mm}^{2}$, and $J_{\mathrm{c}} \sim 600 \mathrm{~A} / \mathrm{mm}^{2}$ overall for $J_{\mathrm{c}}=1000 \mathrm{~A} / \mathrm{mm}^{2}$.

\subsubsection{Cable}

The cabled conductor should carry sufficient current to produce a fast, uniform distribution of the magnet's energy during a quench, so that no region is heated above room temperature. This requirement becomes quite severe for the immense stored energy (several $\mathrm{MJ} / \mathrm{m}$ ) of a $20 \mathrm{~T}$ design. Preliminary calculations indicate that a practical superconducting cable should possibly carry $I \sim 30 \mathrm{kA}$. In addition. the cable must provide local support of the superconductor without exceeding the strain limit for axial as well as for transverse loads beyond which $J_{c}$ is severely degraded (typically $\sim 2 \times 10^{-3}$ for $\mathrm{Nb}_{3} \mathrm{Sn}$ in axial strain and $100 \mathrm{MPa}$ in transverse strain, but even less for some superconductors).

\subsubsection{Structure}

The coil structure must distribute the Lorentz stresses, and the prestresses during assembly, so that the accumulation and concentration of stress nowhere exceeds the strain limits for the superconductor or the materials used for support and insulation. This requirement becomes a particular challenge at $20 \mathrm{~T}$. Extensive knowledge of straininduced reduction and degradation of superconducting materials used is absolutely essential, and a major part of the efforts should focus on the optimization of strain management and overall magnet design.

\subsubsection{Missions and time requirements}

$20 \mathrm{~T}$ class of magnets can certainly not be built within a few years. As pointed out. extensive materials and conductor research and development are required, and have the highest priority. It is believed that when the conductor is available in terms of average current density at $20 \mathrm{~T}$, and the stress levels are foreseen to be manageable, the magnets can be built.

\subsubsection{Message to the high energy physics community}

$20 \mathrm{~T}$ dipole magnets for a future accelerator can be built within a time frame of about 10-15 years, provided the conductors become available. During the past years we have seen a continuous improvement of current density and unit length, in particular of high $T_{c}$ superconductors, to the extent that there is now sufficient confidence that the required current densitics will become available. Thercfore, it would be beneficial if physicists working in the high energy physics community think about the opportunities such a system offers in order to anticipate possible application requirements in the next 10 to 20 years.

\subsubsection{Message to the materials science community}

Enhancement of the critical current density of superconductors at $20 \mathrm{~T}$ is absolutely essential. This could be achieved with improvements of the known materials as well as with presently unknown materials. For the A15 materials, such as $\mathrm{Nb}_{3} \mathrm{Sn}$ and $\mathrm{Nb}_{3} \mathrm{Al}$, artificial fluxpinning at high fields and further shift of $H_{\mathrm{c} 2}$ by optimized doping could be the right approach. For the BSCCO and YBCO family of materials, continuous improvements are reported, but new ways of processing the materials should be investigated and potential methods of technical interest should be recognized and developed in the light of 20 $\mathrm{T} / 1.8 \mathrm{~K}$ applications.

\subsubsection{Message to conductor R\&D groups}

The development of practical conductors and cables for high fields should be stimulated. In a hybrid design both $\mathrm{Nb}_{3} \mathrm{Sn}$ and an advanced conductor, i.e. some form of HTS, will be present. Even with the existing types of high field A15 conductors a further push to higher critical current densities is necessary.

Moreover, in a $20 \mathrm{~T}$ magnet the body forces have to be 
decompled from the local Iorentz forces on the conductor. To attain this decoupling, magnet designers need reinforced strands which are strain optimized cables, or fruitful combinations, to handle the enhanced stress levels in $20 \mathrm{~T}$ class magnets. Conductor R\&D groups are challenged to develop such reinforced conductors $\left(\mathrm{Nb}_{4} \mathrm{Sn}, \mathrm{Nb}_{3} \mathrm{Al}\right.$, BSCCO) which can withstand external transverse stress levels of 200-300 MPa.

\subsubsection{International cooperation and $R \& D$ network}

The number of laboratories involved in the R\&D for future accelerators is limited, and on a world-wide scale only one or two costly high energy physics projects will be approved. The obvious consequence is the need for creating a world-wide network of excellence to carry out the necessary R\&D in the field of superconducting materials, conductors and magnets. The efforts and partial and local funding should be made coherent and amplified to attain the long term goals of this $20 \mathrm{~T}$ project. Sharing of specific expertise, computer codes and testing facilities are essential elements of such a network if it is to succeed.

\section{Acknowledgments}

Portions of this work was supported by the U.S. Department of Energy under contract No. DE-AC0376SF00098. (Appreciation to A. Zichichi et al.)

The authors would like to thank Dr. Antonino Zichichi for his encouragement and support. The work presented was performed at the 29th Eloisatron Workshop sponsored by INFN. We would also like to thank the staff of the "Ettore Majorana" Center for their kind hospitality and assistance during the workshop.

\section{References}

[1] P.F. Dahl, Superconductivity: Its Historical Roots and Development from Mercury to the Ceramic Oxides (American Institute of Physics, New York, 1992).
[2] J.P. Blewett. H. Hahn and A.G. Prodell (eds.), Proc. 1968 Summer Sudy on Superconducting Devices and Accelerators, Brookhaven National Laboratory, BNL-Report 50155 (1969).

[3] P.J. Reardon, IEEE Trans. Magn. MAG-13 (1977) 704.

[4] L. Hoddeson. Historical Studies in the Physical Sciences 18 (1987) 25.

[5] P.F. Dahl, Superconductivity: Its Historical Roots and Development from Mercury to the Ceramic Oxides (AIP, New York, 1992).

[6] P. Wanderer, Proc. 1993 Part. Accel. Conf,. IEEE and American Physical Society (1993) p. 2726.

[7] R. Perin, Particle Accelerator Conf. and Int. Conf, on HighEnergy Accelerators IEEE, Dallas, TX, 1995, Superconducting Mugnets, Vol. 2, p. 1282.

[8] W.A. Barletta and H. Leutz, Supercolliders and Superdetectors, Proc. 19th and 25th Workshop on the INFN Eloisatron Project (World Scientific, Singapore, 1993) pp. 3-42 and 227-233.

[9] P. Schmüser, The Physics of Particle Accelerators, AIP Conf. Proc. 249, eds. M. Month and M. Dienes, vol. 2 (AIP, New York, 1992) p. 1099.

[10] S. Wolf, ibid., p. 1159.

[11] P.M. McIntyre, Proc. 10th Int. Conf. on High Energy Accelerators, Hamburg, July 20-24, 1992, p. 674.

[12] S.L. Wipf and A. Koski, 7th Workshop on Critical Currents in Superconductors, Alpsbach, Austria, 24-27 Jan. 1994.

[13] M.N. Wilson, Superconducting Magnets (Clarendon, Oxford, 1983).

[14] D. Hegedorn and F. Rodriguez-Mateos, IEEE Trans. Magn. MACr-28 (1992) 366 .

[15] ANSYS, a Copyrighted Finite Element Program from Swanson Analysis Systems ANSYS, Inc.

[16] G.E. Gallagher-Daggitt. RIIEL Report M-A25 (1973).

[17] M.D. Sumption et al., CEC/ICMC Conf. Paper TuUPM 2-2. Columbus, $\mathrm{OH}$, July 17-21, 1995.

[18] R. Perin, Particle Accelerator Conf., Dallas, TX, 1995. Superconducting Magnets, p. 1282.

[19] A. Den Ouden et al., Paper AG. 14th Int. Conf. on Magnet Technology, Helsinki, Finland, June 1995.

[20] R.M. Scanlan et al., IEEE Trans. Appl. Superconductivity 5 (1995) 1000.

[21] J.R. Miller et al., IEEE Trans. Magn. 30 (1994) 1563. 